\title{
Trivium
}

Revue franco-allemande de sciences humaines et sociales - Deutsch-französische Zeitschrift für Geistesund Sozialwissenschaften

30 | 2019

La constitution au tournant des XXe et XXle siècles

\section{Die Verfassung an der Wende vom 20. zum}

\section{Jahrhundert}

Eine deutsch-französische Perspektive

\section{Aurore Gaillet}

Traducteur : Olivier Joop

\section{OpenEdition \\ Journals}

Édition électronique

URL : http://journals.openedition.org/trivium/6875

DOI : $10.4000 /$ trivium. 6875

ISSN : $1963-1820$

Éditeur

Les éditions de la Maison des sciences de l'Homme

\section{Référence électronique}

Aurore Gaillet, « Die Verfassung an der Wende vom 20. zum 21. Jahrhundert », Trivium [Online], 30 |

2019, online erschienen am 18 Dezember 2019, abgerufen am 25 September 2020. URL : http:// journals.openedition.org/trivium/6875; DOI : https://doi.org/10.4000/trivium.6875

Ce document a été généré automatiquement le 25 septembre 2020.

\section{(c) (i) (9)}

Les contenus des la revue Trivium sont mis à disposition selon les termes de la Licence Creative Commons Attribution - Pas d'Utilisation Commerciale - Pas de Modification 4.0 International. 


\title{
Die Verfassung an der Wende vom 20. zum 21. Jahrhundert
}

Eine deutsch-französische Perspektive

\section{Aurore Gaillet}

Traduction : Olivier Joop

\begin{abstract}
»Die existentiellen Situationen führen notwendig zu den Grundlagen, während politische Ruhephasen eher die Kultur des geltenden Rechts begünstigten «. ${ }^{1}$
\end{abstract}

1 Was Michael Stolleis hier in Bezug auf das 19. Jahrhundert feststellt, lässt sich ohne weiteres auf die Gegenwart übertragen. Das heute wieder aktuelle Thema der »Vertrauenskrise« der repräsentativen Demokratie ${ }^{2}$ führt auch zu einer Infragestellung der Verfassungsordnungen. Es erscheint daher notwendig, den zentralen Begriff der »Verfassung an der Wende vom 20. zum 21. Jahrhundert« einer Überprüfung zu unterziehen.

2 Vor dem Hintergrund der Feierlichkeiten anlässlich des 70-jährigen Bestehens des Grundgesetzes der Bundesrepublik Deutschland (23. Mai 1949) und des 60-jährigen Bestehens der Verfassung der V. Französischen Republik (4. Oktober 1958) bot es sich förmlich an, eine rechtsvergleichende Bilanz zu ziehen. ${ }^{3}$ Auch das Inkrafttreten der Weimarer Reichsverfassung vor 100 Jahren, am 11. August 1919, sollte dabei nicht vergessen werden. ${ }^{4}$ Derartige Gedenkfeiern bieten Anlass, die Errungenschaften des Konstitutionalismus im Allgemeinen, aber auch die Stabilität der französischen und der deutschen Rechtsordnung im Besonderen zu würdigen. Diese für beide Länder geltende Feststellung darf allerdings nicht darüber hinwegtäuschen, dass zwischen der oftmals als »Erfolgsgeschichte ${ }^{5}$ dargestellten Entwicklung des Grundgesetzes und einer weitaus kritischeren Würdigung der französischen Verfassung ${ }^{6}$ weiterhin ein Graben besteht. Allgemein lässt sich jedoch feststellen, dass die Ausbreitung eines »Verfassungsmodells«, das auf bestimmten formellen und materiellen Kriterien ${ }^{7}$ beruht, zweifelsohne $\mathrm{zu}$ der schrittweisen Überwindung der autoritären und totalitären Regime des 20. Jahrhunderts und zur Festigung des Rechtsstaats 
beigetragen hat. ${ }^{8}$ Andererseits verbietet sich jede blinde Selbstzufriedenheit. ${ }^{9}$ Die zahlreichen auf den Staat einwirkenden Veränderungen, die Erschütterungen der europäischen Demokratien - und ihrer rechtsstaatlichen Dimension -, die Unsicherheiten in Bezug auf die Grundlagen des politischen und sozialen Paktes nunmehr angereichert um eine unverzichtbare ökologische Komponente ${ }^{10}-$, das alles sind Warnzeichen mit Auswirkungen auf diese klassischen Begrifflichkeiten des Verfassungsrechts wie "Staat «" ${ }^{11}$ oder "Verfassung $"$.

3 Die im Zentrum dieses Themenheftes stehende Verfassung ist damit wohl "nicht notwendig an ihr Ende" gekommen..$^{12}$ Jedoch fordern diese "Momente der Verunsicherung ${ }^{13}$ dazu auf, die intellektuelle Auseinandersetzung mit den Begriffen und deren Entwicklungsgeschichte nicht aufzugeben. ${ }^{14}$ »Der geschichtsblinde Jurist ist gefährlich «, ${ }^{15}$ und nur wenn man die Entstehungsbedingungen unserer zentralen Begrifflichkeiten kennt, »kann man weiter fragen, ob sie sich verändert haben und was daraus für Auslegung, Anwendung und Fortentwicklung des geltenden Rechts folgt «. ${ }^{16}$ Ohne Anspruch auf eine erschöpfende Betrachtung - sofern eine solche überhaupt möglich wäre - können die in dieser Nummer versammelten deutschen und französischen Beiträge gemeinsame Überlegungen zum Thema Verfassung anstoßen.

Dabei geht es nicht darum, die Entwicklungen und Veränderungen der beiden gegenwärtig in diesen Ländern geltenden Verfassungen nachzuzeichnen, sondern den Verfassungsbegriff einer historischen, theoretischen und rechtsvergleichenden Betrachtung zu unterziehen (1). Anschließend soll der Blick auf die Verfassungsakteure gerichtet werden (2), womit der dynamische Charakter der Verfassungsordnung betont werden kann, die stets Auslegungen und Konkretisierungen bedarf. Zwar sind Unterschiede zwischen Verfassungstext und Verfassungspraxis unvermeidbar, jedoch stehen normative und politische Auffassung von Verfassung nicht zwangsläufig im Widerspruch zueinander. Ab dem Moment, wo die Verfassung nunmehr in einem zersplitterten politischen und gesellschaftlichen Umfeld gedacht werden muss (3), können diese beiden Bedeutungen sich im Gegenteil sinnvoll ergänzen.

\section{Historische, theoretische und rechtsvergleichende Betrachtung}

5 Oftmals wird hervorgehoben, dass der »verfassungsgeschichtliche Rückblick wie der verfassungsvergleichende Seitenblick [...] dazu prädestiniert [sind], den mit dem eigenen (verfassungsdogmatischen wie) verfassungstheoretischen Standpunkt verbundenen blinden Fleck sichtbar zu machen und kritisch zu beleuchten «. ${ }^{17}$ In Bezug auf die Verfassung wirft eine sowohl historische als auch rechtsvergleichende Analyse, die zugleich rechtstheoretisch und rechtsphilosophisch sein will, das notwendige Licht auf diese Frage.

6 a) Historisch betrachtet, erfährt der Verfassungsbegriff gegen Ende des 18. Jahrhunderts einen tiefgreifenden Bedeutungswandel und überwindet die bislang vorherrschende deskriptive Auffassung der Regierungsformen politischer Gemeinschaften. Der Wandel des Verfassungsparadigmas, in dem staatliche Selbstbehauptung und Souveränität des Volkes zusammentreffen, wird in den Beiträgen von Olivier Beaud und Dieter Grimm im Einzelnen nachgezeichnet. ${ }^{18}$ Die moderne Verfassung mit ihrem Anspruch, eine legitime und durch das Recht begrenzte 
Herrschaft zu errichten - eine Verfassung im nunmehr auch normativen Sinne -, geht aus der amerikanischen und der Französischen Revolution hervor. Die fünf von Dieter Grimm festgestellten Kriterien einer freiheitlichen und demokratischen Verfassung, die das Ergebnis dieses Prozesses der $" N o r m w e r d u n g$ des Verfassungsbegriffes ${ }^{19}$ bildet, verbinden politische und rechtliche Dimensionen, die alle darauf abzielen, den Gedanken einer vorgegebenen, äußeren oder übergeordneten Ordnung zu überwinden: (1) Verfassungen sind Rechtsnormen, die aus einer politischen Willensentscheidung hervorgehen; (2) sie regeln die Einrichtung und die Ausübung der Staatsgewalt; (3) keine anderen Grenzen als diejenigen aus der Verfassung, weder vor- noch außerkonstitutionelle, werden als Begrenzung der Staatsgewalt anerkannt; (4) die Verfassung ist die oberste Rechtsnorm und geht allen anderen rechtlichen Bestimmungen und Regelungen voran; (5) die einzige und ausschließliche Legitimationsquelle der Verfassung ist der Wille des Volkes. ${ }^{20}$

7 b) Rechtstheoretisch betrachtet, können die skizzierten besonderen historischen Entwicklungen nur vor dem Hintergrund der Ideengeschichte, insbesondere der liberalen politischen Philosophie der Aufklärung, verstanden werden. In diesem Sinne besteht eine enge Verknüpfung zwischen Verfassung und Konstitutionalismus ${ }^{21}$ : Die "moderne« Verfassung wird als höchstes niedergeschriebenes Gesetz aus der Feder einer legitimen "verfassungsgebenden Gewalt" (pouvoir constituant) gedacht. Die sich daraus ableitende Unterscheidung zwischen verfassungsgebender Gewalt ${ }^{22}$ und verfassten Gewalten (pouvoirs constitués) ${ }^{23}$ ist hier maßgeblich - die verfassungsgebende Gewalt gehört zur Macht des Volkes, im Gegensatz namentlich zu den alten, von den Fürsten oktroyierten Verfassungen (monarchische »Souveränität« bzw. Legitimität). Gemäß der doppelten Stoßrichtung von Artikel 16 der Erklärung der Menschen- und Bürgerrechte gewährleistet die freiheitliche und demokratische Verfassung die »(Grund-)Rechte« des Einzelnen und legt die "Trennung der Gewalten ${ }^{24}$ fest. Sie geht im Übrigen einher mit dem Prozess fortschreitender Unterscheidung zwischen Privatsphäre und öffentlichem Raum. ${ }^{25}$

8 c) Eine rechtsvergleichende Betrachtung erlaubt schließlich eine noch genauere Analyse. Alle hier vorgestellten Beiträge unterstreichen die Bedeutung der unterschiedlichen Rechtstraditionen, der sozialen Rahmenbedingungen und der Denkgewohnheiten. So hat sich die Entwicklung in Deutschland während des 19. Jahrhunderts abgehoben vom klassischen liberalen Konstitutionalismus. ${ }^{26} \mathrm{Nach}$ dem Zusammenbruch des »III. Reiches« hat eine neue Verfassungskultur - man sprach sogar von einem "Verfassungspatriotismus ${ }^{27}$ - die Wiedererrichtung des Rechtsstaats begleitet; dabei wurde ein besonderer Akzent auf die Gerichtsbarkeiten und die Grundrechte $^{28}$ gelegt. In Frankreich hat die revolutionäre Durchsetzung der Prinzipien des Konstitutionalismus von 1789 das Land nicht vor »den Unbilden des französischen Konstitutionalismus « bewahrt: die gesetzes-zentristische Tradition und das "republikanische« Misstrauen gegenüber der rechtsprechenden Gewalt haben die freiheitliche Verfassungskultur in gewissem Sinne "geschwächt « ${ }^{29}$ Ungeachtet feststellbarer Gemeinsamkeiten der "europäischen Verfassungsordnungen «, ${ }^{30}$ wie beispielsweise die schrittweise Herausbildung einer »deutsch-französischen« oder gar einer europäischen ${ }^{31}$ Rechtsgemeinschaft, prägen diese Traditionen noch immer das nationale Rechtsdenken dies- und jenseits des Rheins. ${ }^{32}$ 


\section{Die Verfassung und ihre Akteure}

\section{a) Normative Verfassung, Verfassungsgerichtsbarkeit und Konstitutionalisierung des Rechts}

Sobald die Verfassung als Rechtsnorm begriffen wird, stellt sich die Frage nach den Verfahren zu deren wirksamer Durchsetzung. Die Sicherung der Verfassung erfordert zwar nicht zwangsläufig einen gerichtlichen Schutz, jedoch wird der Aufstieg des Konstitutionalismus immer mehr mit der Einrichtung einer Verfassungsgerichtsbarkeit in Zusammenhang gebracht. ${ }^{33}$

Die beiden hier übersetzten Artikel von Louis Favoreu und Helmuth Schulze-Fielitz betonen diesbezüglich das heutige Vorherrschen einer normativen Dimension von Verfassung, die mit den »Grundrechten und -freiheiten« verknüpft ist und von einer "Konstitutionalisierung des Rechts« verstärkt wird; diese wird ihrerseits von den Verfassungsgerichten alimentiert. In Frankreich war die Verrechtlichung des Verfassungsrechts von einem Teil der Rechtslehre bereits lange vor dem sogenannten " nouvel élan« (neuen Aufschwung) (A. Viala) gefordert worden, der durch Einführung des neuen prozessrechtlichen Instruments der vorrangigen Frage zur Verfassungsmäßigkeit (question prioritaire de constitutionnalité - QPC) im Jahr 2010 ausgelöst worden war. Der Leitartikel der ersten Ausgabe der 1990 von Louis Favoreu ins Leben gerufenen Revue française de droit constitutionnel ist in dieser Hinsicht programmatisch zu verstehen.

11 Die Unterschiede zum deutschen Verfassungsmodell sind dennoch erheblich, denn der Verfassungsrat war ursprünglich nicht als Gericht zur Wahrung der Grundrechte und Grundfreiheiten konzipiert worden. Der Kontrast zwischen den beiden Systemen bleibt offensichtlich, sowohl in Bezug auf die Zusammensetzung als auch die Zuständigkeiten der jeweiligen Verfassungsgerichte. ${ }^{34}$ Davon zeugt auch in den hier veröffentlichten Beiträgen die Hervorhebung zunächst der sehr weitreichenden Kompetenzen des deutschen Bundesverfassungsgerichts, darunter eine dem französischen Recht fremde Urteilsverfassungsbeschwerde (M. Jestaedt), sodann der besonders dynamischen Grundrechtsjudikatur ${ }^{35}$ des Gerichts - namentlich seit dem Lüth-Urteil vom 15. Januar 1958, das H. Schulze-Fielitz aus der Retrospektive, 50 Jahre danach, analysiert. Andreas Voßkuhle, Präsident des Bundesverfassungsgerichts (2010-2020) wirft seinerseits einen erhellenden Blick auf die bedeutsamen, vom Bundesverfassungsgericht gesetzten Impulse für eine (auch europäische) »Integration durch Recht«.

\section{b) Welche Rolle für die Verfassungsrechtslehre?}

Die beiden Beiträge von Matthias Jestaedt und Alexandre Viala laden zu einer näheren Betrachtung der Rolle der Rechtslehre ein. Der Artikel des Ersteren zeigt auf, wie tiefgreifend die »Dogmatik« die deutsche Rechtskultur prägt. ${ }^{36}$ In ihrer die Anwendung und Systematisierung des positiven Rechts begleitenden Funktion ist diese Dogmatik in Deutschland weitaus präziser ausgestaltet als in Frankreich. Dazu gesellt sich eine »fast schon symbiotische" Synergie zwischen Rechtslehre und Rechtspraxis, die aufgrund der Bedeutung der Rechtsprechung des Bundesverfassungsgerichts besonders das deutsche Verfassungsrecht charakterisiert (M. Jestaedt). Die deutsche Rechtslehre, mit ihren in einer weit zurückreichenden und ruhmreichen Rechtstradition liegenden 
Wurzeln, ${ }^{37}$ bleibt ein »starker Partner« der Gerichte. Doch mahnen die deutschen Juristen ständig, sich nicht mit dieser »Partnerrolle« zu begnügen und damit ihren Diskurshorizont zugunsten eines "Bundesverfassungsgerichts-Positivismus « 38 einzuschränken und die Tradition der Systematisierung der Dogmatik zu vernachlässigen.

Es überrascht nicht, dass ähnliche Warnungen vor der "Gefahr einer Unterordnung « ${ }^{39}$ einer lediglich die Verfassungsrechtsprechung paraphrasierenden Rechtslehre auch in Frankreich zu hören sind. Wie Alexandre Viala hervorhebt, liefe eine im Wesentlichen auf die Rechtsprechung des Verfassungsrates fokussierte und dieser nachplappernde Rechtslehre Gefahr, lediglich einem in der Verwaltungsrechtslehre seit langem betretenen Pfad zu folgen, ohne jedoch dabei Impulse von einer ähnlich mächtigen Institution wie dem Staatsrat zu erhalten. Mehr noch: ein Vergleich mit dem deutschen Bundesverfassungsgericht schärft die Kritik an den Schwächen des französischen Verfassungsrates. ${ }^{40}$ In Bezug auf die französische Rechtslehre weist die Kritik an der "Überbetonung des Verfassungsprozesses« (A. Viala) darüber hinaus in eine andere Richtung. Der Fokus liegt dabei nicht so sehr auf der »Dogmatik« - einem Begriff, der im französischen Verfassungsrecht selten verwendet wird beziehungsweise diesem "fremd" ist ${ }^{41}$-, sondern drückt sich vornehmlich in einer stets neuen erkenntnistheoretischen Suche mit dem Ziel aus, eine "wahre" Wissenschaft vom Verfassungsrecht anerkennen zu lassen. Darüber hinaus ist es bedeutsam, im Rahmen dieser Suche auch die Gegenstände des Verfassungsrechts zu erkennen: den prozessrechtlichen und den politischen Aspekt dieses Bereiches auszuformulieren, bleibt für die Rechtswissenschaft eine stimulierende Herausforderung. ${ }^{42}$

\section{c) Staatsorganisationsrecht und demokratische Wachsamkeit}

In Frankreich wie in Deutschland sind Stimmen zu hören, die eine zu "positivistische« Lesart der Verfassung kritisieren. Sie werde von einer "Grundrechtsbesessenheit « ${ }^{43}$ genährt, welche ihrerseits das demokratische System schwäche. Die Aufforderung, »die Wirklichkeit der Welt des Rechts hinter der Silhouette der Richter « zu entdecken (A. Viala) oder die Rolle des Rechts im europäischen Integrationsprozesses zu begrüßen (A. Voßkuhle), darf nicht dazu führen, die politische Dimension der Verfassung zu verschleiern. Diese Dimension kann sich als besonders notwendig herausstellen, wenn die Fragmentierung des Staates im Besonderen und der politischen Macht im Allgemeinen sowie das Auseinanderbrechen des sozialen Gefüges das Nachdenken über die Verfassung schwieriger gestalten.

\section{Das Verfassungsdenken in einem fragmentierten Umfeld}

Da der Begriff der Verfassung mit der Geschichte des Staates verwoben ist, darf die Frage nach den Transformationen des Staates selbst ${ }^{44}$ und deren »Auswirkungen auf die Verfassung ${ }^{45}$ nicht ausgeklammert werden. 


\section{a) Verfassung und Staat im Angesicht des Prozesses der Europäisierung und der Internationalisierung} neue Nahrung gegeben, welche die Grenzen eines staatszentrierten Ansatzes des Verfassungsrechts unterstreichen, einschließlich in Deutschland, dem bundesstaatliche Strukturen doch eigentlich vertraut sind. ${ }^{47}$ Vor diesem Hintergrund legen Constance Grewe und Hélène Ruiz-Fabri in ihrem Beitrag dar, inwiefern der Rückgriff auf das Verfassungsvokabular ebenso wie die diesen Rückgriff begleitenden »erbitterten« Auseinandersetzungen das atypische Wesen des europäischen Einigungsprozesses veranschaulichen. Zweifellos erfuhren diese Fragen eine besondere Resonanz bei der Ausarbeitung des »Vertrages über eine Verfassung für Europa« (2004). Das Scheitern dieses Vertrages im Ratifizierungsprozess (2005) und die Verabschiedung eines klassischen Vertragswerks (Vertrag von Lissabon, 2009) haben allerdings keinen Schlusspunkt unter diese Debatten gesetzt. Wird nicht einerseits die »Verfassungsidentität ${ }^{48}$ der Staaten gegen eine manchmal als zu stark vereinheitlichend empfundene europäische Integration ins Feld geführt? Sucht die Europäische Union andererseits nicht selbst nach einer eigenen »Identität ${ }^{49}$ und stützt sich dabei auf eine »Wertegemeinschaft", die auf einem von den nationalen Verfassungsrichtern und den europäischen Richtern bekräftigten und geteilten Verfassungsinstrumentarium beruht (Voßkuhle) $?^{50}$

18 Von einem rechtsvergleichenden Standpunkt aus ist die insbesondere seit dem Lissabon-Urteil des Bundesverfassungsgerichts ${ }^{51}$ verstärkte Vitalität des deutschen Rechtsdiskurses hervorzuheben. Ungeachtet der seit 1949 für das Grundgesetz charakteristischen "offenen Staatlichkeit «52 und der Abgrenzung vom klassischen Begriff der »Souveränität«, lässt sich diese Vitalität auch dadurch erklären, dass das Grundgesetz außergewöhnliche Verfassungsbestimmungen enthält (z. B. die »Ewigkeitsgarantie« nach Art.79, Abs. 3 GG oder auch die Bestimmung über die Geltungsdauer des Grundgesetzes in Art.146 GG) und dass das Bundesverfassungsgericht eine ausgesprochen dynamische Judikatur verfolgt. Sein Präsident Andreas Voßkuhle manifestiert offen den Willen des Gerichts, aktiv an der Entwicklung des europäischen Rechts mitzuwirken. ${ }^{53}$ Ohne Zweifel hat nicht nur Deutschland diese Absicht. Doch sind die Bereiche außerordentlich breit, zu denen die »Impulse« aus Deutschland kommen. Man denke an die Schlagwörter »Grundrechte« (vgl. dazu die Frage des europäischen Haftbefehls ${ }^{54}$ ), Verfassungsidentität ${ }^{55}$ (vgl. dazu die Rechtsprechung des EuGH zum kirchlichen Arbeitsrecht ${ }^{56}$ ) oder »ultra vires« (vgl. dazu die sensible Frage der Politik der EZB $^{57}$ ).

19 Die Konstitutionalisierung des Völkerrechts und die Diskurse über den "globalen Konstitutionalismus ${ }^{58}$ bieten gleichfalls Perspektiven für eine eingehendere Betrachtung des Verfassungsbegriffes jenseits des Staates. Allerdings finden sie ihre Grenzen in ihrer Uneinheitlichkeit und in noch nicht abgeschlossenen Entwicklungen. Von dieser Feststellung ausgehend, darf bezweifelt werden, ob der »Bedeutungsverlust, den die Verfassung auf der nationalen Ebene erleidet, auf der internationalen [oder der europäischen] Ebene kompensiert werden kann« (Grimm). Derartige Zweifel bilden 
andererseits die Grundlage für Untersuchungen, die ein verstärktes, zumindest aber beständiges Interesse am Staat erkennen lassen. ${ }^{59}$

\section{b) Verfassung und Gesellschaft im Kontext der neuen Erwartungen an den Staat}

Letztendlich bleibt festzuhalten, dass, gleichgültig wie groß die Unwägbarkeiten auch sein mögen, denen die französische und die deutsche Verfassungsordnung ausgesetzt sind, das Fortbestehen dieser Verfassungsordnungen sowohl Flexibilität und Bewahrung ihrer Identität als auch Entwicklung und Stabilität voraussetzt. In diesem Zusammenhang stellt sich die Frage, unter welchen Bedingungen der demokratisch verfasste Staat weiterhin den Rahmen des sozialen Gefüges bilden kann. Die wiederholten Bemühungen, den Bürger "präsenter« zu machen, ihm »neuartige Rechte« zu geben oder auch ihn ins "Zentrum des Institutionengefüges« zu stellen, ${ }^{60}$ sind kein Zufall. Stärkung der repräsentativen Demokratie als identitätsstiftendes Band zwischen Regierenden und Regierten, ${ }^{61}$ Ausweitung der partizipativen Demokratie, insbesondere durch eine tatsächliche Mitwirkung des Wirtschafts-, Sozial- und Umweltrates (Conseil économique, social et environnemental) oder dessen Umgestaltung in eine ganz andere "auf Langfristigkeit « ${ }^{62}$ angelegte Kammer; Stärkung der Rolle des Bürgers als Grundstein des Gesellschaftsvertrages - all diese unterschiedlichen Pfade gilt es miteinander zu verbinden und zu beschreiten.

Bereits die frühen politischen Denker warfen die Frage auf nach dem Verhältnis von Demokratie und Freiheitlichkeit, nach der Gefahr einer Tyrannei durch die Mehrheit ${ }^{63}$ sowie nach der Möglichkeit einer »wirksamen« Ausübung der Souveränität durch das Volk. ${ }^{64}$ Desgleichen wurden die Unzulänglichkeiten der Grundannahmen liberaler Denker und der Unterscheidung zwischen Privatsphäre und öffentlichem Raum insbesondere in Bezug auf die Ausweitung der Aufgaben des Sozialstaats (und der zunehmenden Bedeutung der wirtschaftlichen Akteure) ${ }^{65}$ - bereits sehr früh thematisiert.

Die Überlegungen über den Verfassungsbegriff an der Wende vom 20. zum 21. Jahrhundert über die hier zusammengestellten Beiträge hinaus weiterzuführen, setzt voraus, diesen Fragestellungen vertieft nachzugehen, denn die Fortentwicklung einer zunehmend pluralen Gesellschaft führt zu neuartigen Erwartungen nicht nur an die politische Macht, sondern auch an deren verfassungsrechtliches Fundament.

\section{BIBLIOGRAPHIE}

Balladur, É. (Hg.) (2007): Une V Vépublique plus démocratique: rapport du comité de réflexion et de proposition sur la modernisation et le rééquilibrage des institutions de la $V^{e}$ République, Paris: La Documentation française. 
Baranger, D. (2003): „Constitutionnalisme«, in: Alland, D. / Rials, S. (Hg.): Dictionnaire de la culture juridique, Paris: PUF, S. 266-271.

Baranger, D. / Beaud, O. (2008): »Présentation de la revue«, in: Jus Politicum, Nr. 1 [verfügbar unter http://juspoliticum.com/article/Presentation-de-la-revue-24.html].

Bartolone, C. / Winock, M. (Hg.) (2015): Refaire la démocratie. Dix-sept propositions, Paris: éd. Thierry Marchaisse.

Beaud, O. (1993): »La souveraineté de l'État, le pouvoir constituant et le Traité de Maastricht«, in: RFDA, S. 1045-1068.

Beaud, O. (1994): La puissance de l'État, Paris: PUF.

Beaud, O. (1998): »Fédéralisme et souveraineté. Notes pour une théorie de la Fédération«, in: RDP, Nr. 1, S. 83-122.

Beaud, O. / Heyen, E. V. (1999): Eine deutsch-französische Rechtswissenschaft? - Une science juridique franco-allemande? Kritische Bilanz und Perspektiven eines kulturellen Dialogs - Bilan critique et perspectives d'un dialogue culturel, Baden-Baden: Nomos.

Beaud, O. (2003): »Doctrine«, in: Alland, D. / Rials, S. (Hg.): Dictionnaire de la culture juridique, Paris: PUF, S. 384-388.

Beaud, O. / Conte, P. / Wachsmann, P. (2018): »Préface«, in: Jus Politicum, Nr. 20-21 (»La jurisprudence du Conseil constitutionnel et les différentes branches du droit«), S. 5-6.

Böckenförde, E.-W. (1961): Die deutsche verfassungsgeschichtliche Forschung im 19. Jahrhundert, Berlin: Duncker \& Humblot.

Böckenförde, E.-W. (1972): »Der Verfassungstyp der deutschen konstitutionellen Monarchie im 19. Jahrhundert «, in: Böckenförde, E.-W. / Wahl, R.: Moderne deutsche Verfassungsgeschichte (18151918), Köln: Kiepenheuer und Witsch, S. 146-170.

Böckenförde, E.-W. (1976 [1969]): »Entstehung und Wandel des Rechtsstaatsbegriffs «, in: ders.: Staat, Gesellschaft, Freiheit. Studien zur Staatstheorie und zum Verfassungsrecht, Frankfurt a. M.: Suhrkamp, S. 65-92.

Böckenförde, E.-W. (1976 [1972]): »Die Bedeutung der Unterscheidung von Staat und Gesellschaft im demokratischen Sozialstaat der Gegenwart«, in: ders.: Staat, Gesellschaft, Freiheit. Studien zur Staatstheorie und zum Verfassungsrecht, Frankfurt a. M.: Suhrkamp, S. 185-220.

Böckenförde, E.-W. (1991 [1983]): »Demokratie und Repräsentation. Zur Kritik der heutigen Demokratiediskussion «, in: ders.: Staat, Verfassung, Demokratie. Studien zur Verfassungstheorie und zum Verfassungsrecht, Frankfurt a. M.: Suhrkamp, S. 379-405.

Bogdandy, A. v. (2011): »La science juridique dans l'espace juridique européen, une réflexion à partir de l'exemple allemand«, in: Recueil Dalloz, Nr. 41, S. 2816-2822.

Bonin, P. / Brunet, P. / Kerneis, S. (Hg.) (2018): Formes et doctrines de l'État, Paris: Pedone.

Bourg, D. (Hg.) (2017): Inventer la démocratie du XXI' siècle. L'Assemblée citoyenne du futur, Paris: Éditions les liens qui libèrent.

Bourg, D. / Whiteside, K. (2010): Vers une démocratie écologique. Le citoyen, le savant et le politique, Paris: Seuil.

Carré de Malberg, R. (2003 [1919/22]): Contribution à la théorie générale de l'État, Paris: Dalloz.

Chagnollaud, D. (Hg.) (2008): Les 50 ans de la Constitution, 1958-2008, Paris: Litec. 
Di Fabio, U. (2018): Die Weimarer Verfassung. Aufbruch und Scheitern, München: Beck.

Dreier, H. (2009): Gilt das Grundgesetz ewig? Fünf Kapitel zum modernen Verfassungsstaat, München: Carl-Friedrich-von-Siemens-Stiftung.

Dreier, H. / Waldhoff, C. (Hg.) (2018): Das Wagnis der Demokratie. Eine Anatomie der Weimarer Reichsverfassung, 2. Aufl., München: Beck.

Evans, P. B. / Rueschemeyer, D. / Skocpol, T. (Hg.) (2010 [1985]): Bringing the State Back in, Cambridge: Cambridge University Press.

Gaillet, A. (2012): L'individu contre l'État. Étude sur l'évolution des recours de droit public dans l'Allemagne du XIX ${ }^{e}$ siècle, Paris: Dalloz.

Gaillet, A. / Perlo, N. / Schmitz, J. (2018): La confiance. Un dialogue interdisciplinaire, Toulouse: Presse de l'Université Toulouse 1 Capitole.

Gaillet, A. / Hochmann, T. / Marsch, N. / Vilain, Y. / Wendel, M. (2019): Droits constitutionnels français et allemand. Une perspective comparée, Issy-les-Moulineaux: L.G.D.J.

Gaillet, A. / Herrera, C. M. (Hg.) (2020): Weimar. Réflexions autour d'une Constitution centenaire. Actes du colloque de Cergy-Pontoise, Paris: PUF (Revue Française de Droit Constitutionnel), im Erscheinen. Gauchet, M. (2017): L'avènement de la démocratie, Bd. 4, Le nouveau monde, Paris: Gallimard.

Gerkrath, J. (1998): L'émergence d'un droit constitutionnel pour l'Europe. Modes de formation et sources d'interprétation de la Constitution des Communautés et de l'Union européenne, Brüssel: Éditions de l'Université de Bruxelles.

Gosewinkel, D. (2018): »The Constitutional State«, in: Pihlajamäki, H. / Dubber, M. D. / Godfrey, M.: The Oxford Handbook of European Legal History, Oxford: Oxford University Press, S. 945-973.

Grewe, C. / Ruiz-Fabri, H. (1995): Droits constitutionnels européens, Paris: PUF.

Grimm, D. (1987): Die Zukunft der Verfassung, Frankfurt a. M.: Suhrkamp.

Grimm, D. (2012a): »Die Errungenschaft des Konstitutionalismus und ihre Aussichten in einer veränderten Welt«, in: ders.: Die Zukunft der Verfassung II, Frankfurt a. M.: Suhrkamp, S. 315-344.

Grimm, D. (1989): „Verfassung II«, in: Brunner, O. /, Conze, W. / Koselleck, R. (Hg.): Geschichtliche Grundbegriffe. Historisches Lexikon zur politisch-sozialen Sprache in Deutschland, Stuttgart: Klett-Cotta, Bd. 6, S. $863 \mathrm{ff}$.

Grimm, D. (1999): „Das Grundgesetz nach 50 Jahren - Versuch einer staatsrechtlichen Würdigung ", in: Bundesministerium des Inneren (Hg.): Bewährung und Herausforderung. Die Verfassung vor der Zukunft, Wiesbaden: VS Verlag für Sozialwissenschaften, S. 39-59.

Grimm, D. (2001): „Verfassungspatriotismus nach der Wiedervereinigung «, in: ders.: Die Verfassung und die Politik, München: Beck, S. 107-117.

Grimm, D. (2012b): »Types of Constitutions«, in: Rosenfeld, M. / Sajö, A. (Hg.): The Oxford Handbook of Comparative Constitutional Law, Oxford: Oxford University Press, S. 98-132.

Grimm, D. (2018): „Nachdenken über Verfassung in den Bielefelder Jahren«, in: Davy, U. / LübbeWolff, G. (Hg.): Verfassung: Geschichte, Gegenwart, Zukunft. Autorenkolloquium mit Dieter Grimm, Baden-Baden: Nomos, S. 13-33.

Grimm, D. (2019a): »Auf Bewährung«, in: FAZ, 9. Januar 2019. 
Grimm, D. (2019b): „Wie sähe heute ein neues Grundgesetz aus?«, in: Heinig, H. M. / Schorkopf, F. (Hg.): 70 Jahre Grundgesetz. In welcher Verfassung ist die Bundesrepublik?, Göttingen: Vandenhoeck \& Ruprecht, S. 287-288.

Gusy, C. (2018): 100 Jahre Weimarer Verfassung. Eine gute Verfassung in schlechter Zeit, Tübingen: Mohr Siebeck.

Habermas, J. (1987): »Über den doppelten Boden des demokratischen Rechtsstaates«, in: ders.: Eine Art Schadensabwicklung, Frankfurt a. M.: Suhrkamp, S. 18-23.

Herrera, C. M. (2012): „Variation, évolution, métamorphose: les significations du concept de constitutionnalisme«, in : Pouderon, B. / Casas, J. (Hg.): Variation, évolution, métamorphose, SaintEtienne: Presses Universitaires, S. 359-370.

Herrera, C. M. (2015): Le Constitutionnalisme latino-américain aujourd'hui: entre renouveau juridique et essor démocratique?, Paris: Kimé.

Hochmann, T. (2019): „Droits fondamentaux «, in: Gaillet, A. / Hochmann, T. / Marsch, N. / Vilain, Y. / Wendel, M. (2019): Droits constitutionnels français et allemand. Une perspective comparée, Issy-lesMoulineaux: L.G.D.J., S. 367-420.

Huber, E. R. (1988): Deutsche Verfassungsgeschichte seit 1789, Bd. 2: Der Kampf um Einheit und Freiheit, 1830 bis 1850, 3. Aufl., Stuttgart: Kohlhammer.

Huber, P. M. (1993): Maastricht - Ein Staatsstreich?, Stuttgart: R. Boorberg.

Hummel, J. / Heuschling, L. (2006): »Le libéralisme du Vormärz, la figure du professeur politique«, in: Revue française d'Histoire des Idées Politiques, Nr. 24, S. 227-231.

Jestaedt, M. (2010): »Verfassungstheorie als Disziplin«, in: Depenheuer, O. / Grabenwarter, C. (Hg.): Verfassungstheorie, Tübingen, Mohr Siebeck, S. 3-56.

Jouanjan, O. (1996): »La forme républicaine du gouvernement, norme supraconstitutionnelle?«, in: Mathieu, B. / Verpeaux, M. (Hg.): La République en droit français, Paris: Economica, S. 267-287.

Jouanjan, O. (2000): »Présentation«, in: Böckenförde, E.-W.: L'État, le droit et la constitution démocratique. Essais de théorie juridique, politique et constitutionnelle, Paris: L.G.D.J.

Jouanjan, O. (Hg.) (2001): Figures de l'État de droit. Le Rechtsstaat dans l'histoire intellectuelle et constitutionnelle de l'Allemagne, Straßburg: Presses Universitaires de Strasbourg.

Jouanjan, O. (2003): „Ce que donner une Constitution à l'Europe veut dire«, in: Cités, Nr. 13, S. 2135.

Jouanjan, O. (2005): Une histoire de la pensée juridique en Allemagne, 1800-1918, Paris: PUF.

Jouanjan, O. / Masing, J. (Hg.) (2011): Verfassungsgerichtsbarkeit. Grundlagen, Innerstaatliche Stellung, Überstaatliche Einbindung, Tübingen: Mohr Siebeck.

Klein, C. (1996): Théorie et pratique du pouvoir constituant, Paris: PUF.

Kotzur, M. (2011): »Deutschland und die internationalen Beziehungen - >offene Staatlichkeit nach 60 Jahren Grundgesetz«, in: JöR, Nr. 59, S. 389-409.

Le Divellec, A. (2011): »Jus Politicum. Le droit ressaisi par la politique«, in: nonfiction.fr. [verfügbar unter https://www.nonfiction.fr/articleprint-4108-

jus_politicum__le_droit_ressaisi_par_la_politique_htm]. 
Le Pillouer, A. (2005-2006): »Pouvoir constituant originaire et pouvoir constituant dérivé: à propos de l'émergence d'une distinction conceptuelle«, in: Revue d'histoire des facultés de droit et de la science juridique, Nr. 25-26, S. 123-141.

Lepsius, O. (2008): »Themen einer Rechtwissenschaftstheorie«, in: Jestaedt, M. / Lepsius, O. (Hg.): Rechtswissenschaftstheorie, Tübingen: Mohr Siebeck, S. 1-50.

Levitsky, S. / Ziblatt, D. (2018 [2017]): Wie Demokratien sterben: Und was wir dagegen tun können, Übersetzung von Klaus-Dieter Schmidt, München: Deutsche Verlags-Anstalt.

Manent, P. (2012): Histoire intellectuelle du libéralisme, Paris: Hachette.

Marsch, (2019): »Justice constitutionnelle«, in: Gaillet, A. / Hochmann, T. / Marsch, N. / Vilain, Y. / Wendel, M. (2019): Droits constitutionnels français et allemand. Une perspective comparée, Issy-lesMoulineaux: L.G.D.J., S. 315-366.

Mathieu, B. (Hg.) (2008): Cinquantième anniversaire de la Constitution française, Paris: Dalloz.

Mathieu, B. (2017): Le droit contre la démocratie?, Issy-les-Moulineaux: L.G.D.J.

Mayer, F.C. (2003): »La Charte européenne des droits fondamentaux et la Constitution européenne«, in: RTDE, S. $175 \mathrm{ff}$.

Mehdi, R. (2015): »L'identité de l'Union européenne«, in: Levade, A. / Michel, V. / Stefanini, M. / Mehdi, R.: L'identité à la croisée des États et de l'Europe: Quel sens? Quelles fonctions?, Brüssel: Bruylant, S. $143-161$.

Meinel, F. (2019): Vertrauensfrage. Zur Krise des heutigen Parlamentarismus, München: Beck.

Merten, D. (1992): „Verfassungspatriotismus und Verfassungsschwärmerei«, in: Verwaltungsarchiv, Nr. 83, S. $283 \mathrm{ff}$.

Millet, F.-X. (2013): L'Union européenne et l'identité constitutionnelle des États membres, Paris: L.C.D.G.

Mohnhaupt, H. (1989): „Verfassung I«, in: Brunner, O. /, Conze, W. / Koselleck, R. (Hg.): Geschichtliche Grundbegriffe. Historisches Lexikon zur politisch-sozialen Sprache in Deutschland, Stuttgart: Klett-Cotta, Bd. 6, S. $831 \mathrm{ff}$.

Mohnhaupt, H. / Grimm, D. (2002 [1995]): Verfassung. Zur Geschichte des Begriffs von der Antike bis zur Gegenwart, Berlin: Duncker \& Humblot.

Möllers, C. (2008): Der vermisste Leviathan. Juristische Staatstheorie in der Bunderepublik, Frankfurt a. M.: Suhrkamp.

Möllers, C. (2009): „Verfassungsgebende Gewalt - Verfassung - Konstitutionalisierung. Begriffe der Verfassung in Europa«, in: Bogdandy, A. v. / Bast, J. (Hg.): Europäisches Verfassungsrecht. Theoretische und dogmatische Grundzüge, 2. Aufl., Berlin: Springer.

Möllers, C. (2011): Staat als Argument, 2. Aufl., München: Beck.

Müller, J. W. (2010): Verfassungspatriotismus, Berlin: Suhrkamp.

Murswiek, D. (1993): »Maastricht und der Pouvoir Constituant«, in: Der Staat, Nr. 2, S. 161 ff.

Oppermann, T. (2009): »Deutschland in guter Verfassung? - 60 Jahre Grundgesetz«, in:

JuristenZeitung, Nr. 10, S. 481-491.

Ost, F. (1995): La nature hors la loi. L'écologie à l'épreuve du droit, Paris: La Découverte.

Perlo, N. (2018): „Du dualisme au pluralisme, la protection intégrée des droits fondamentaux en Italie«, in: J. Andriantsimbazovina (Hg.): Intégration et Droits de l'homme, Paris: Mare \& Martin, S. 179-196. 
Pernice, I. (2007): »Theorie und Praxis des Europäischen Verfassungsverbundes«, in: Calliess, C. (Hg.): Verfassungswandel im europäischen Staaten- und Verfassungsverbund. Göttinger Gespräche zum deutschen und europäischen Verfassungsrecht, Tübingen: Mohr Siebeck, S. 61-92.

Rivero, J. (1969): „La protection des droits de l'homme dans les rapports entre personnes privées«, in: Cassin, R.: Amicorum Discipulorumque Liber, Paris: Pédone, Bd. 3, S. 311-322.

Romano, S. (2015): »La crise de l'État moderne« (ins Französische übertragen von D. Soldini), in: Jus Politicum, Nr. 14. [Verfügbar unter http://juspoliticum.com/article/L-Etat-moderne-et-sacrise-968.html].

Rousseau, J. J. (2011 [1762]): Vom Gesellschaftsvertrag oder Grundsätze des Staatsrechts, in Zusammenarbeit mit Eva Pietzcker übersetzt und herausgegeben von Hans Brockard, Stuttgart: Reclam.

Schlink, B. (1989): „Die Entthronung der Staatsrechtswissenschaft durch die Verfassungsgerichtsbarkeit«, in: Der Staat, Nr. 28, S. 161-172.

Schlink, B. (2007): »Abschied von der Dogmatik. Verfassungsrechtsprechung und Verfassungsrechtswissenschaft im Wandel«, in: JuristenZeitung, Nr. 62, S. 157-162.

Schönberger, C. (2014): „Bundesverfassungsgerichtspositivismus: Zu einer Erfolgsformel Bernhard Schlinks«, in: Nolte, J. / Poscher, R. / Wolter, H. (Hg.): Die Verfassung als Aufgabe von Wissenschaft, Praxis und Öffentlichkeit: Freundesgabe für Bernhard Schlink zum 70. Geburtstag, Heidelberg et al.: Müller, S. 41-49.

Sommermann, K.-P. (2008): »Offene Staatlichkeit: Deutschland«, in: Bogdandy, A. v. / Cruz Villalon, P. / Huber, P. M.: Handbuch Ius Publicum Europaeum, Bd. 2, § 14, Heidelberg: Müller.

Sternberger, D. (1979): „Verfassungspatriotismus«, in: FAZ, 23. Mai.

Sternberger, D. (1990 [1982]): »Verfassungspatriotismus«, in: Schriften, Bd. X, Frankfurt a. M. / Leipzig: Insel.

Stolleis, M. (1992): Geschichte des öffentlichen Rechts in Deutschland, Bd. 2: 1800-1914, München: Beck. Stolleis, M. (1990): »Rechtsstaat«, in: Erler, A. / Kaufmann, E. (Hg.): Handwörterbuch zur Deutschen Rechtsgeschichte, 1971-1998, Bd. 4, Berlin: Erich Schmidt Verlag, S. 367-375.

Stolleis, M. (1996): »Der geschichtsblinde Jurist ist gefährlich«, in: $F A Z$, 23. Januar.

Tocqueville, A. de (1984 [1835]): Über die Demokratie in Amerika, herausgegeben von Jacob P. Mayer in Gemeinschaft mit Theodor Eschenburg und Hans Zbinden, übersetzt von Hans Zbinden, beide Teile in einem Band, München: Deutscher Taschenbuch Verlag.

Tourard, H. (2000): L'internationalisation des constitutions nationales, Paris: L.G.D.J.

Troper, M. (2001): „Sur l'usage des concepts juridiques en histoire«, in: ders.: La théorie du droit, le droit, l'État, Paris: PUF.

Troper, M. (2008): »Identité constitutionnelle«, in: Mathieu, B. (Hg.): Cinquantième anniversaire de la Constitution française, Paris: Dalloz, S. 123-131.

Vedel, G. (Hg.) (1993): Propositions pour une révision de la Constitution: rapport au Président de la République, Paris: La Documentation française.

Vogel, K. (1964): Die Verfassungsentscheidung des Grundgesetzes für eine internationale Zusammenarbeit, Tübingen: Mohr Siebeck. 
Voßkuhle, A. (2016): »IIntegration durch Recht « - Der Beitrag des Bundesverfassungsgerichts«, JuristenZeitung, Nr. 71 (4), S. 161-168.

\section{NOTES}

1. Stolleis (1992), S. 423.

2. Gaillet et al. (2018); Meinel (2019). Für eine Betrachtung dieser Frage jenseits des deutschfranzösischen Rechtsraums, vgl. beispielsweise Levitsky / Ziblatt (2018 [2017]).

3. Hierzu s. ebenfalls das Kapitel »Perspektives« (Kap. 9. A. Gaillet) im Sammelband von Gaillet et al. (2019). Die vorliegende Einführung greift Auszüge aus dem genannten Kapitel auf.

4. Gusy (2018); Di Fabio (2018); Dreier / Waldhoff (2018). Siehe dazu auch Gaillet / Herrera (2020).

5. Oppermann (2009), S. 481 ff. und insbesondere S. 491. Auch die weiteren runden Jahrestage des Inkrafttretens des Grundgesetzes haben Anlass zu ähnlichen Kommentaren gegeben. So wurde in Karlsruhe am 23. Mai 2019 ein »Verfassungsfest« gefeiert, begleitet von einer Diskussionsrunde zum Thema "70 Jahre Grundgesetz - Deutschland in guter Verfassung?«. Eine Erweiterung der Perspektive bieten u. a. Herrera (2012) und (2015).

6. Zahlreiche Beiträge anlässlich des 50-jährigen Bestehens der Verfassung von 1958 wiesen diesbezüglich bereits vielsagende Titel auf und betonten immer wieder bestehende Ungleichgewichte und unklare Regelungen in Bezug auf das Verhältnis der Gewalten. Vgl. insbesondere Chagnollaud (2008); Mathieu (2008). Anlässlich des 60-jährigen Bestehens der Verfassung von 1958 scheint diese eher Gegenstand fragender Betrachtungen zu sein, die den Blick auf den "Sinn« (Titre VII, Les Cahiers du Conseil constitutionnel, Nr. 1, 2018) der Verfassung richten, »neue Blickwinkel« (Pouvoirs, Nr. 166, 2018) zu eröffnen suchen, beziehungsweise zehn Jahre nach der Verfassungsreform von 2008 Bilanz ziehen wollen (RFDC 2018/4).

7. Beispielhaft kann der Begriff der "Verfassung" klassischerweise definiert werden als »Gesamtheit der höchsten Normen einer Rechtsordnung, die nur gemäß einem bestimmten Änderungsverfahren novelliert werden dürfen und deren Vorrang wirksam durch ein Gericht sichergestellt wird. Diese Normen regeln die Organisation, die Gestalt und die Struktur eines demokratisch verfassten Rechtsstaats, bestimmen die Organe des Staates sowie deren Beziehungen zueinander und deren Zuständigkeiten und legen die Rechtsstellung des Bürgers im Staatswesen fest, einschließlich deren unveräußerlicher Rechte«, Gerkrath (1998), S. 51.

8. Böckenförde (1976 [1969]); Jouanjan (2001); Stolleis (1990), S. $367 \mathrm{ff.}$

9. Vgl. bereits Grimm (1999), S. 39 ff.; Dreier (2009), S. 102. Vgl. nunmehr Grimm (2019a); (2019b),

S. $287 \mathrm{f}$.

10. Bourg / Whiteside (2010). Vgl. auch Ost (1995).

11. Beaud (1994).

12. Grimm (2012a), S. 330 (franz. Übersetzung in dieser Nummer enthalten, § 35).

13. Voßkuhle (2016), S. 161 (franz. Übersetzung in dieser Nummer enthalten, §1).

14. Troper (2001).

15. Stolleis (1996).

16. Grimm (2018), S. 28.

17. Jestaedt (2010), S. 24 f.

18. Dazu s. auch Mohnhaupt (1989) und Grimm (1989), S. 831 f. bzw. S. 863 f. Ausführlicher Mohnhaupt / Grimm (2002 [1995]); Grimm (1987), S. 221 ff.; Gosewinkel (2018), S. 945 ff.

19. Grimm (2012b).

20. Grimm (2012b), insbesondere. S. 104.

21. Baranger (2003), S. $266 \mathrm{ff}$. 
22. Carré de Malberg (2003 [1919/22]), Teil II, Kap. IV, »Du pouvoir constituant«; Klein (1996); Gosewinkel (2018); Möllers (2009).

23. Le Pillouer (2005-2006), S. 123 ff. Für eine kritische Sichtweise s. Jouanjan (1996), S. 267 ff.

24. Artikel 16 der Erklärung der Menschen- und Bürgerrechte vom 26. August 1789 besagt: „Eine Gesellschaft, in der die Verbürgung der Rechte nicht gesichert und die Gewaltenteilung nicht festgelegt ist, hat keine Verfassung «. Diesbezüglich sei angemerkt, dass im deutschen Sprachraum der Begriff der "Gewaltenteilung« dem im Französischen verwendeten Terminus der "Gewaltentrennung" (séparation des pouvoirs) vorgezogen wird. $\mathrm{Zu}$ diesem Thema s. den Grundsatzbeschluss des Bundesverfassungsgerichts vom 30. Juni 2015, BVerfGE 139, 321 (361).

25. Zu dieser grundsätzlichen Frage s. Böckenförde (1976 [1972]).

26. Zu den Haupttendenzen des deutschen Liberalismus im 19. Jahrhundert vgl. Huber (1988), S. 371 f.; Böckenförde (1961), S. 92 f.; für eine genauere Analyse: Böckenförde (1972). Für Beiträge in französischer Sprache vgl. Hummel / Heuschling (2006), S. 227 ff.; Gaillet (2012).

27. Dieser Begriff findet sich in der deutschen Rechtsdiskussion erstmals im Zuge der Feierlichkeiten anlässlich des 30-jährigen Bestehens des Grundgesetzes: Sternberger (1979) und (1990 [1982]). Namentlich durch ihre Verwendung durch Jürgen Habermas (1987, S. 19) ist diese Wendung in den Sprachgebrauch übergegangen. S. auch Grimm (2001), S. 107 ff.; Müller (2010). Für eine kritische Würdigung s. Merten (1992), S. $283 \mathrm{f}$.

28. Mayer (2003), S. 175 f. und insbesondere S. 178 (hier zitiert v. Grewe und Ruiz-Fabri) spricht an dieser Stelle sogar von einer »Besessenheit« (»obsession«) der deutschen Rechtsprechung und Rechtslehre in Bezug auf die Grundrechte.

29. Baranger (2003), S. 268.

30. Grewe / Ruiz-Fabri (1995).

31. Beaud / Heyen (1999); v. Bogdandy (2011), S. 2818.

32. Hier wäre eine vertiefende Analyse der Besonderheiten des jeweiligen Verfassungsrechts und Verfassungsdenkens sehr sinnvoll. Bezüglich des Verfassungsrechts vgl. beispielsweise Gaillet u. a. (2019).

33. Jouanjan / Masing (2011).

34. Marsch (2019).

35. Hochmann (2019).

36. Lepsius (2008), S. 16.

37. Jouanjan (2005).

38. Schönberger (2014), S. 41 ff.; Schlink (1989), S. 161 ff.; ders. (2007), S. 157 ff.

39. Beaud (2003), S. 384 ff. und insb. S. 388.

40. Beaud et al. (2018).

41. Hochmann (2019).

42. In diesem Sinne s. Baranger / Beaud (2011); Le Divellec (2011).

43. Mayer (2003), S. 178. In Frankreich nehmen kritische Beiträge zu diesem Thema in der letzten Zeit wieder zu: Gauchet (2017); Mathieu (2017).

44. Romano (2015).

45. Grimm (2018), S. 28.

46. S. bereits Huber (1993); Beaud (1993); Murswiek (1993), S. 163.

47. Jouanjan (2003), S. 23 f.; Beaud (1998), S. 89.

48. Troper (2008), S. 123 ff.; Millet (2013).

49. Mehdi (2015), S. $143 \mathrm{ff}$.

50. Hierzu s. auch Pernice (2007), S. $61 \mathrm{ff}$.

51. Urteil vom 30. Juni 2009 (BVerfGE 123, 267 - Lissabon).

52. Vogel (1964); Sommermann (2008); Kotzur (2011), S. 389 ff.

53. Vgl. insbesondere Italienischer Verfassungsgerichtshof, ordinanza $n^{\circ} 24 / 2017$ und Urteil $\mathrm{n}^{\circ}$ 115/2018 im Rahmen der Rechtsache Taricco (EuGH, Gr. Kammer, 8. September 2015, Taricco, 
Rs. C-105/14; und EuGH, Gr. Kammer, 5. Dezember 2017, M.A.S und MB, Rs. C-42/17); dazu Perlo (2018).

54. BVerfGE 140, 317 und EuGH, verb. Rs. C-404/15 und C-659/15 PPU (Aranyosi u. a.), ECLI :EU :C : $2016:$ :198.

55. Zur traditionellen Rechtsprechung des BVerfG siehe BVerfGE 73, 339 [375].

56. EuGH, Rs. C-414/16 (Egenberger), ECLI :EU :C :2018:257. Siehe demgegenüber die herkömmliche Rechtsprechung des BVerfG : BVerfGE 137, 273.

57. BVerfGE 142, 123, nach EuGH, Rs. C-62/14 (Gauweiler), ECLI :EU :C :2015:400; BVerfGE 146, 216 und EuGH, Rs. C-493/17 (Weiss u. a.), ECLI :EU :C :2018:1000.

58. Tourard (2000). S. auch die dieser Frage gewidmete Ausgabe Nr. 19 der Zeitschrift Jus Politicum.

59. Evans et al. (2010); Beaud (1994); Möllers (2011) und (2008); Bonin et al. (2018).

60. S. Vedel (1993) bzw. Balladur (2007) und Bartolone / Winock (2015).

61. Jouanjan (2000), S. 34. Zur repräsentativen Demokratie s. Böckenförde (1991 [1983]).

62. Bourg (2017).

63. Constant, Principes de politique (1815); Tocqueville (1984 [1835]). Für einen Gesamtüberblick, s. Manent (2012).

64. Rousseau (1762). Für eine Einordnung in einen größeren Zusammenhang, s. beispielsweise die Beiträge zum »allgemeinen Willen« in: Jus Politicum (2014), Nr. 10.

65. S. z. B. Rivero (1969), S. $311 \mathrm{ff.}$

\section{INDEX}

Mots-clés : introduction, constitution

Schlüsselwörter : Einleitung, Verfassung

\section{AUTEURS}

\section{AURORE GAILLET}

Aurore Gaillet ist Professorin für öffentliches Recht an der Universität Toulouse 1 Capitole. Nähere Informationen finden Sie hier. 